\title{
A Feedback-based Print Quality Improving Strategy for FDM 3D Printing: An Optimal Design Approach
}

\author{
Tariku Sinshaw Tamir D - Gang Xiong (D - Qihang Fang • \\ Xisong Dong (D) - Zhen Shen* (D) - Fei-Yue Wang (D)
}

Received: date / Accepted: date

\begin{abstract}
Fused deposition modelling (FDM) 3D printing, as a supporting technology in social manufacturing and cloud manufacturing, is a rapidly growing technology in the era of industry 4.0. It produces objects with the layer-by-layer material accumulation technique. However, qualitative uncertainties are the common challenges yet. In order to assure print quality, studying the error causing parameters and minimizing their effects is important. This paper presents a feedback-based error compensation strategy, which integrates fuzzy inference system and grey wolf optimization algorithm. The objectives are twofold. First, the possible errors in FDM 3D printing are discussed in detail and optimal error causing parameters are obtained in percentage. This is used to understand the effects of the printing errors in every phase of the 3D printing process. From the nine optimization configuration trials used, Config6 that has 100 number of iterations and 60 wolves is
\end{abstract}

Tariku Sinshaw Tamir and Qihang Fang

The State Key Laboratory for Management and Control of Complex Systems, Beijing Engineering Research Center of Intelligent Systems and Technology, Institute of Automation, Chinese Academy of Sciences, Beijing 100190, China and also with the School of Artificial Intelligence, University of Chinese Academy of Sciences, Beijing 100049, China

E-mail: tamir@ia.ac.cn, fangqihang2020@ia.ac.cn

Gang Xiong, Xisong Dong, Zhen Shen and Fei-Yue Wang The State Key Laboratory for Management and Control of Complex Systems, Beijing Engineering Research Center of Intelligent Systems and Technology, Institute of Automation, Chinese Academy of Sciences, Beijing 100190, China and also with Guangdong Engineering Research Center of 3D Printing and Intelligent Manufacturing, Cloud Computing Center, Chinese Academy of Sciences, Dongguan 523808, China

E-mail: gang.xiong@ia.ac.cn, xisong.dong@ia.ac.cn, zhen.shen@ia.ac.cn, feiyue.wang@ia.ac.cn

* Corresponding author selected due to its higher convergence speed and best fitness value. The integral absolute error (IAE) is used as an objective function and the global minimum is achieved in the iteration interval $[86,100]$. The outputs of this optimization problem is used to achieve the next objective. Second, a closed-loop quality monitoring approach comprising of inner-loops and an outer-loop is utilized. The three inner-loops are used to monitor the errors during pre-printing, printing, and post-printing, respectively. The outer-loop, on the other hand, is responsible for monitoring the aggregated errors in all the three 3D printing phases. The error compensation system simulation in Matlab is run for 10 seconds, and the results show that the "normal" range deformation factors are reached within less than 2 seconds for the inner-loops, whereas the outer-loop deformation factor is achieved within 7 seconds. The responses are within the acceptable time range.

Keywords Industry $4.0 \cdot 3 \mathrm{D}$ printing · Print quality . Error causing parameters - Deformations - Fuzzy inference system

\section{Introduction}

The advancement of science and technology is improving people's life quality. The mass production of commodities is becoming uncompetitive with the personalized market. The individual interest oriented commodities are favored by the customers. Three dimensional (3D) printing, as a massively distributed manufacturing (MDM), is a viable solution for customer interest-based fabrication [1. 3D printing possesses the following steps 2. It starts from computer aided design (CAD) modelling. Then, the CAD model gets into a certain slicing algorithm to generate printing toolpath. Based on the 


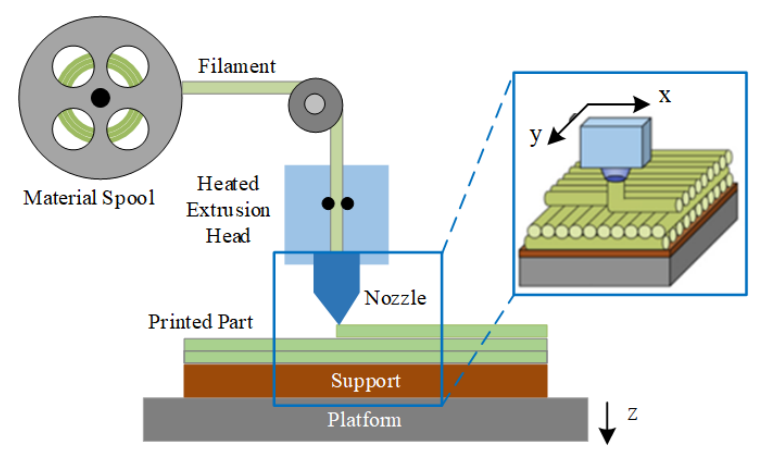

Fig. 1 FDM 3D printing

defined toolpath, the part building is done. The last step is post-processing in which the printed part is further enhanced by the removal of support structures. An arbitrary shape can be produced by fused-depositionmodelling (FDM) 3D printing, schematically shown in Fig. 1. FDM 3D printing, as a supporting technology in social manufacturing and cloud manufacturing, is a rapidly growing technology. It produces objects with a layer-by-layer material accumulation technique [3,4]. In FDM, the thermoplastic filament is moved from the extruder cold end to hot end, where it is heated until molten. Then the filament in molten state is fed through the nozzle to accumulate on the heated build platform with layer-by-layer fashion until the finished part is produced. The advantages of FDM include simple technology, affordable system cost, and the ability to create complicated shapes and recommended manufacturing time.

However, FDM 3D printing is suffering difficulties in controlling errors during a phenomenon, like, nozzle clogging, material runout, excessive vibration, defects associated with cooling and heating, and under extrusion or over extrusion [5,6]. Problems affecting the quality of 3D prints are also reported in [7]. Those factors reduce the reliability of FDM technique that fundamentally affect the quality of the printed part. Surface roughness, porosity, geometric deviation, and poor interconnection between layers [8,9] are some of the $3 \mathrm{D}$ printing inaccuracies, which result in print quality issue. Optimization with regard to production speed, product accuracy and development cost is an active research area in $3 \mathrm{D}$ printing. This paper presents a mechanism to enhance print quality by incorporating a closed-loop control framework in 3D printing. An endto-end feedback-based error compensation mechanism is proposed to minimize the errors associated with every phase of the $3 \mathrm{D}$ printing process. Modelling the $3 \mathrm{D}$ printing process mathematically, as a subset of digital twin technology, is the primary task before diving into modelling and optimization of a control framework. Digital twin is a virtual representation of physical systems in an attempt to high-fidelity modelling and simulations. It monitors, simulates, diagnoses, predicts, and controls the formation process and behavior of products in the real environment. The first definition of digital twin was made by Michael Grieves in 2003 for presenting product life cycle management (PLM). Its definition in its original form was stated as a digital representation of physical system, and emerged as an entity on its own or some way of linkage with the physical system [10]. Rolle et al. 11] implemented a digital twin architecture focusing on industry 4.0. This industrial revolution is considered as a new technological wave transforming industrial environment dramatically. Fuller et al. [12 wrote a review paper on digital twin enabling technologies and their challenges in the three application domains: manufacturing, healthcare and smart cities.

The rest of the paper elucidates the errors associated with every phase of the $3 \mathrm{D}$ printing process and proposes an end-to-end error compensation strategy. Section 2 presents the researchers' perspectives on mechanisms for improving print quality, and also introduces the use of control systems and optimization algorithms in industries. Section 3 outlines the detailed description of the types of errors in every phase of 3D printing. Section 4 proposes an end-to-end 3D printing error compensation strategy. Section 5 presents results and discussions, followed by conclusions and perspectives in section 6 .

\section{Related work}

Most of the existing 3D printers lack automatic error compensation and quality monitoring mechanism. Therefore, nowadays, researchers are giving attention to minimize the effects of 3D printing errors for assuring print quality. Various types of error compensation strategies are reported in the literature. The following paragraph presents a detailed report on data-driven print quality monitoring system in FDM 3D printing.

Liu et al. 13 proposed an image-based closed-loop quality monitoring system for fused filament fabrication $(\mathrm{FFF})$, in which the quality issues are mitigated via online processing parameter adjustment. Similarly, Faes et al. 14 integrated a two-dimensional laser scanner into a $3 \mathrm{D}$ printer machine, and monitored the process state variables online to improve the accuracy of printing. Ikeuchi et al. [15] developed a data-efficient neural network model in cold spray additive manufacturing to predict the geometry of the printed part. The proposed model was also used for modelling of other depositionbased additive manufacturing technologies. Saluja et al. 
[16] proposed a convolutional neural network (CNN)based warping detection system, which captures each of the print layers and extracts the corners, and the extracted region of interest is then fed to a CNN model to monitor the printing process. Cerro et al. 17 also developed a machine learning model to predict surface roughness of printed parts manufactured by using FDM $3 \mathrm{D}$ printer. Moreover, in-situ monitoring of FFF is reviewed [18,19], which then derives the FFF technology to the next generation of systems by enabling robust closed-loop control scheme in 3D printing.

Most of the aforementioned research works follow component-wise (i.e., application-specific) quality monitoring strategy to enhance the quality of the $3 \mathrm{D}$ printing process. Thus, those print quality monitoring approaches may not probably consider and generalize all the factors that affect the performance of the $3 \mathrm{D}$ printer. The quality of the printed object is affected by every phase of the $3 \mathrm{D}$ printing process. The objective of this paper is to present an end-to-end quality monitoring framework for minimizing the effects of the 3D printing errors and further improving the quality of the printed object. It considers the errors associated in pre-printing, printing and post-printing. A feedback-based fuzzy inference system (FIS) along with the grey wolf optimization (GWO) algorithm is used for the error compensation framework. The following paragraphs present a detailed report on applications of FIS and GWO for controlling and optimization task in industries.

Fuzzy logic was introduced by Lotfi A. Zadeh in 1965 [20. Since then it has been applied to model imprecise, linguistic and uncertain data [21,22]. Unlike conventional control scheme, whose controlling performance highly depends on the modelling accuracy of physical system, fuzzy inference engine is convenient when analytical model is difficult to get while expert experience is available. Thus, it is a viable solution to extend the controlling capability of fuzzy system in manufacturing industry where analytical model is missing while expert knowledge is available. Li et al. [23] proposed a fuzzy multi-criteria modelling used in service-oriented manufacturing for the problem of fuzzy scheduling. Similarly, Wang et al. 24] used a dynamic adaptive fuzzy system to evaluate the reliability of manufacturing system with multiple production lines.

The GWO algorithm is first coined by Mirjalili et al. [25]. It is a kind of meta-heuristic-based optimization method that mimics the special hunting behavior of a group of grey wolves living together. Mirjalili and his co-workers tested the performance of GWO algorithm with 29 well known functions and reported that the algorithm provided very competitive performance compared to the other meta-heuristic optimization meth- ods. Ghorpade et al. 26] used GWO technique in automotive industry to position wireless sensor nodes optimally in the parking area for vehicle parking system. Similarly, Yan et al. 27] magnified the performance of GWO algorithm by benchmarking 23 widely used test functions over a known engineering design problem.

\section{An end-to-end error analysis in FDM 3D printing}

Before we go deep into the error compensation strategy of an FDM 3D printing, it is very essential to discuss the sources of errors associated with its printing process. The main sources of errors are observed in the three phases: pre-printing phase, printing phase, and post-printing phase. Beyond the aforementioned error sources, the $3 \mathrm{D}$ printing process is also affected by some external disturbances that result in printing error. The summed up errors are reflected in the quality of the printed object. The general description of errors in every phase of the $3 \mathrm{D}$ printing process is illustrated in Fig. 2.

\subsection{Pre-printing phase}

File format conversion error and slicing error are the two known causes of printing errors under this stage. The surface of 3D model is represented by STereoLithography (STL) file format with small triangles. But, this file format is not the exact representation of the model, which then results in some error in the printed object. Cao and Miyamoto 28] proposed a direct slicing algorithm which precisely slices the $3 \mathrm{D}$ CAD model and reduces the errors caused by STL file conversion. Similarly, Feng et al. 29] developed a direct slicing algorithm for T-spline surfaces. Their work designs T-spline surface and calculates the slicing points on the surface. It then achieves better accuracy and higher manufacturing efficiency.

Slicing of the 3D model is the core and the very important task in the $3 \mathrm{D}$ printing process. Layer height is one of the slicing parameter that plays a major role for assuring the quality of the printed object. The thinner the layers are, the better the quality of the object will be. But, this results in lower printing speed. On the contrary, thicker layer yields higher printing speed. But, it leads to more steps in the printed object that reduces the surface quality. To create an optimal trade-off between fabrication time and surface quality, Mao et al. 30] proposed an adaptive slicing method to generate an efficient slicing plan. The proposed algorithm is based on dynamic programming and searches 


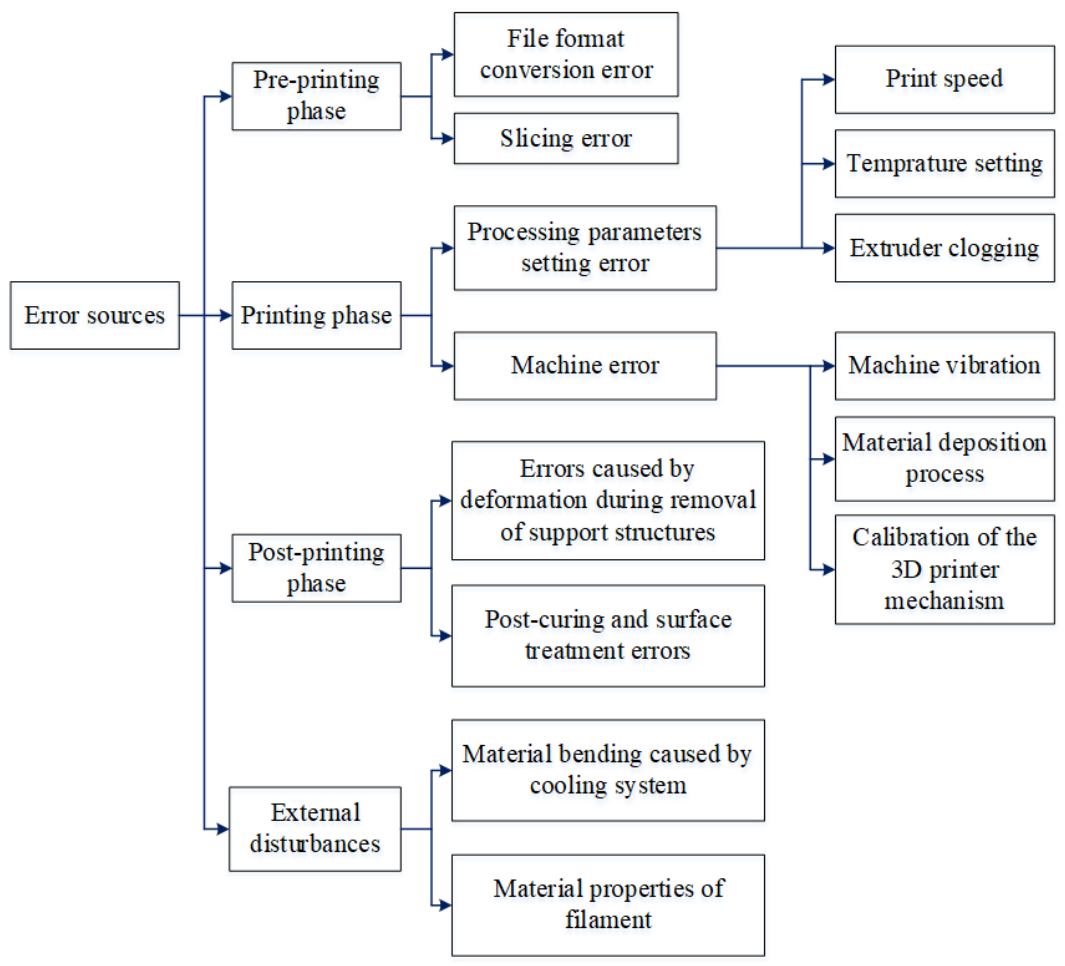

Fig. 2 Source of errors in FDM 3D printing

for the best printing direction. Similarly, Garashchenko and Zubkova 31] designed an adaptive slicing algorithm which adopts variable layer height by considering the angle between the surface of the object and the printing direction. Their algorithm can then reduce the printing time.

\subsection{Printing phase}

Processing parameters setting errors and machine errors are the major causes of printing errors in this stage. Processing parameters setting plays a crucial role for assuring the quality of the printed object. Optimal processing parameters may reduce printing errors and then enhance print quality. Print speed, print bed temperature, and extruder temperature are some of the main parameters that affect the quality of the $3 \mathrm{D}$ printing process 32. Improper setting of those parameters may lead to extruder clogging, under extrusion, over extrusion, etc., which severely affects the material deposition process, resulting in poor print quality. Zhang et al. 33. proposed a method to predict surface roughness in extrusion-based additive manufacturing by considering three processing parameters such as, layer thickness, extruder temperature and print speed. Similarly, Khanzadeh et al. 34 presented a machine learning-based porosity prediction scheme from the thermal history of melt-pool for direct laser deposition. Frick 35] also discussed about how to avoid errors during desktop 3D printing.

We consider three factors to study the machine errors. They can be listed as machine vibration, material deposition process, and 3D printer calibration. The 3D printer under run condition may experience vibration on its components that result in loss of the printing path. This is a severe issue that affects the structural geometry of the print object resulting in print quality problem 36. Another major factor to appear error in the $3 \mathrm{D}$ printing process is during material deposition. The air gap between the print head of the extruder and the print bed must be in optimal distance. If the gap is large it may result in loss of contact between each of the layers, whereas the lower gap may cause deformations. Therefore, the proper calibration of $3 \mathrm{D}$ printer components prior to the printing action is needed.

\subsection{Post-printing phase}

Traditional planar slicing requires support structures to print overhangs and complex parts of the object [37. After the completion of 3D printing process, support structures need to be removed from the main part of the object. However, the quality of the printed part is significantly affected through the removal process. Zhao et 
al. 38] proposed a nonplanar slicing method for robotic additive manufacturing. Their algorithm attempts to print 3D object without using support structures which results in improved quality of the printed part as compared to objects printed with planar slicing algorithm. Similarly, Ahlers et al. 39] used nonplanar slicing to minimize discretization effects in additive manufacturing process. Their work proposed a novel slicing algorithm for FDM 3D printing that combines planar and nonplanar layers, resulting in stronger and smoother object surfaces and increased printing quality.

\subsection{External disturbances}

One major problem in 3D printing is the bending of the printed part through cooling operation. Untimely cooling system may lead the material to bend in one direction that results in printed part quality issue [40]. An appropriate and timely cooling mechanism should be supplied into the $3 \mathrm{D}$ printing process in the time of material deposition. Thus, each of the printing layer gets stick well with its successive layer and the strength of the final product can be guaranteed layer by layer.

Another factor on the quality of the printed part is the material properties of the filament. Metals, alloys, polymers, composites, bio-materials, ceramics, and concrete are the main known materials to be used in $3 \mathrm{D}$ printing. Thermoplastic polymers such as polyamide (PA), acrylonitrile-butadiene-styrene copolymers (ABS), polylactic acid (PLA) and polycarbonate (PC) are mainly in the form of filaments for FDM 3D printing [41. The composite of polymers with fibers enhances the mechanical properties of the printed parts to be used as functional components and load-bearings [42,43, 44].

\section{The proposed error compensation strategy}

\subsection{Modelling of FDM 3D printer and control system design}

The overall mathematical representation of an end-toend FDM 3D printer with the incorporation of feedback system is shown in Fig. 3. It tries to give knowledge about the use of closed-loop-based quality monitoring in the domain of FDM 3D printing.

A feedback-based control system is designed for the compensation of errors to enhance the print quality in FDM 3D printer. The proposed error compensation framework is shown in Fig. 4. It consists of a control system, a model of 3D printer and an optimization algorithm. The control system comprises of inner-loops and an outer-loop, which represents the whole control framework. There are three inner-loops having three FIS control schemes, FIS-1, FIS-2, and FIS-3, respectively. Those individual inner-loops control approaches are dedicated to analyze the effects of error causing parameters in each phase of the $3 \mathrm{D}$ printing process. The outer-loop, on the other hand, is used to compensate the summed-up effects of errors by deploying another FIS scheme in hope of fitting the actual model with the target model.

We considered three conditions for analyzing the effects of errors on the print quality. The followings are the representations of error causing parameters, which can be evaluated in percentage. 1) During pre-printing phase, denoted by $\left.P_{1}, 2\right)$ during printing phase, denoted by $P_{2}$, and 3 ) during post-printing phase, denoted by $P_{3}$. Error $(E)$ and Integral of Error $(I E)$ are fed to FIS, whereas the enlargement or reduction of the new model is the output from FIS. This applies for all FIS structures. Error is defined as the difference between the target model and the actual model. Therefore, the formation of the new model is a function of $P_{1}, P_{2}, P_{3}$, $E$ and $I E$, and it is expressed as:

$$
\left[\begin{array}{l}
M_{1}^{*} \\
M_{2}^{*} \\
M_{3}^{*} \\
M^{*}
\end{array}\right]=\left[\begin{array}{l}
f\left(P_{1}, E_{1}, I E_{1}\right) \\
f\left(P_{2}, E_{2}, I E_{2}\right) \\
f\left(P_{3}, E_{3}, I E_{3}\right) \\
f\left(P_{1}, P_{2}, P_{3}, E, I E\right)
\end{array}\right]
$$

where $M_{1}{ }^{*}, M_{2}{ }^{*}$ and $M_{3}{ }^{*}$ are the new model representations of the three inner-loops respectively, and $M^{*}$ is the new model representation of the outer-loop.

The proposed Multi Input Single Output (MISO) FIS takes "Error" and "Integral of Error" as inputs and outputs the new model deformation factors as shown in Fig. 5. A trial-and-error procedure is applied to determine the range of fuzzy membership functions. A Mamdani type fuzzy inference system [45] is used. The FIS operations are as follows. 'Min' for 'And', 'Min' for 'Implication', 'Max' for 'Aggregation', 'Centroid' for 'Defuzzification' and triangular membership functions, are used. The twenty-five IF...THEN structured fuzzy rules are designed from the two linguistic input variables. Each has five linguistic values. Fig. 5 presents the overall FIS structure that shows the interconnection of each linguistic values to form a set of fuzzy rules.

Linear-fitting, which is a universal modelling technique involving less amount of calculation, results better printing accuracy as compared with non-liniear-fitting modelling technique [6]. Thus, we intend to use it to represent the mapping between the new model and the actual model in FDM 3D printer. It is mathematically written as:

$y=\alpha x+\beta$ 


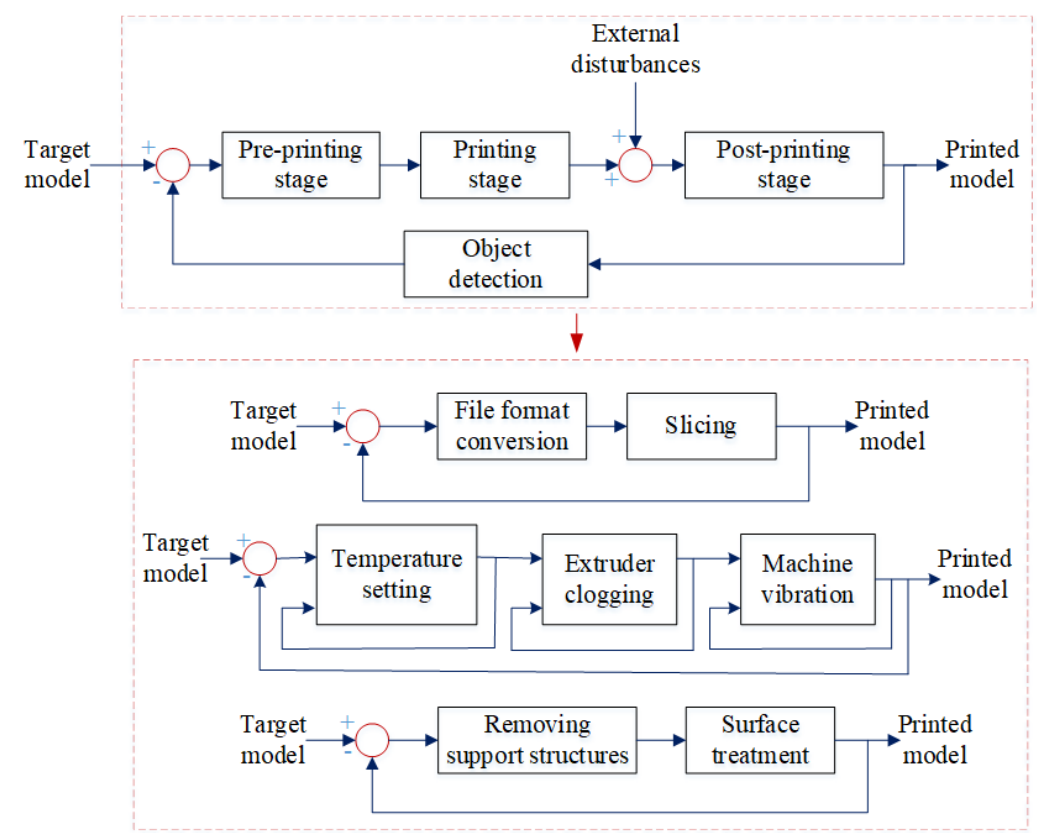

Fig. 3 The mathematical modelling of FDM 3D printer

where $y$ is the actual model size and $x$ is the new model size. The two model sizes are the average value of the three points of the data at different positions of each side length. This kind of measurement is useful to consider the printed object inaccuracies of size, shape and printing position. The terms $\alpha$ and $\beta$ are the two deformation factors. The aim is to find the best function matching between $x$ and $y$. The new model versus the actual model relationships for the three individual inner-loops are expressed as:

$$
\begin{aligned}
& y_{1}=\alpha_{1} x+\beta_{1} \\
& y_{2}=\alpha_{2} x+\beta_{2} \\
& y_{3}=\alpha_{3} x+\beta_{3}
\end{aligned}
$$

Thus, the sum of the output of the three inner-loops is fed to the outer-loop's FIS to master control the error compensation problem.

\subsection{Optimization method}

The GWO algorithm is applied to determine the optimal solutions for the three error causing parameters, $P_{1}, P_{2}$, and $P_{3}$. This algorithm is a meta-heuristic-based optimization method with the inspiration of social hierarchy of the grey wolves 25. Grey wolves have a habit of living, hunting, and eating together in their hierarchical order. The hierarchy's top level is alpha $(\alpha)$ wolf that monitors and leads the whole pack and decides the time to walk, hunt, sleep, and so on. Whereas, the hierarchy's second level is beta $(\beta)$ wolf that helps $\alpha$ wolf for decision making. The hierarchy's lowest level is omega $(\omega)$ wolf. The other category of wolf, on the other hand, which is neither $\alpha$-wolf, $\beta$-wolf, nor $\omega$-wolf is known as delta $(\delta)$ wolf. The grey wolves' hunting process comprises encircling and attacking.

The mathematical expression of encircling during the hunting process is given as:

$\mathbf{D}=\left|\mathbf{C} \cdot \mathbf{X}_{P}(t)-\mathbf{X}(t)\right|, \quad \mathbf{C}=2 \cdot \mathbf{r}_{1}$

$\mathbf{X}(t+1)=\mathbf{X}_{p}(t)-\mathbf{A} \cdot \mathbf{D}, \quad \mathbf{A}=2 \mathbf{m} \cdot \mathbf{r}_{2}-\mathbf{m}$

where $\mathbf{D}$ is the distance from the prey to the grey wolves, $\mathbf{X}_{P}$ and $\mathbf{X}$ are the position vectors of the prey and the grey wolf, respectively, and $t$ is the current iteration. The terms $\mathbf{r}_{1}$ and $\mathbf{r}_{2}$ are the two random numbers in the range $[0,1]$. The term $\mathbf{m}$ is a decreasing vector with components from 2 to 0 . The knowledge of $\alpha, \beta$, and $\delta$ wolves' position is used to compute the potential location of the prey. The wolves' position updating algorithm is governed as:

$$
\begin{aligned}
& \mathbf{D}_{\alpha}=\left|\mathbf{C}_{1} \cdot \mathbf{X}_{\alpha}-\mathbf{X}\right| \\
& \mathbf{D}_{\beta}=\left|\mathbf{C}_{2} \cdot \mathbf{X}_{\beta}-\mathbf{X}\right| \\
& \mathbf{D}_{\delta}=\left|\mathbf{C}_{3} \cdot \mathbf{X}_{\delta}-\mathbf{X}\right| \\
& \mathbf{X}_{1}=\mathbf{X}_{\alpha}-\mathbf{A}_{1} \cdot \mathbf{D}_{\alpha} \\
& \mathbf{X}_{2}=\mathbf{X}_{\beta}-\mathbf{A}_{2} \cdot \mathbf{D}_{\beta} \\
& \mathbf{X}_{3}=\mathbf{X}_{\delta}-\mathbf{A}_{3} \cdot \mathbf{D}_{\delta} \\
& \mathbf{X}(t+1)=\frac{\mathbf{X}_{1}+\mathbf{X}_{2}+\mathbf{X}_{3}}{3}
\end{aligned}
$$




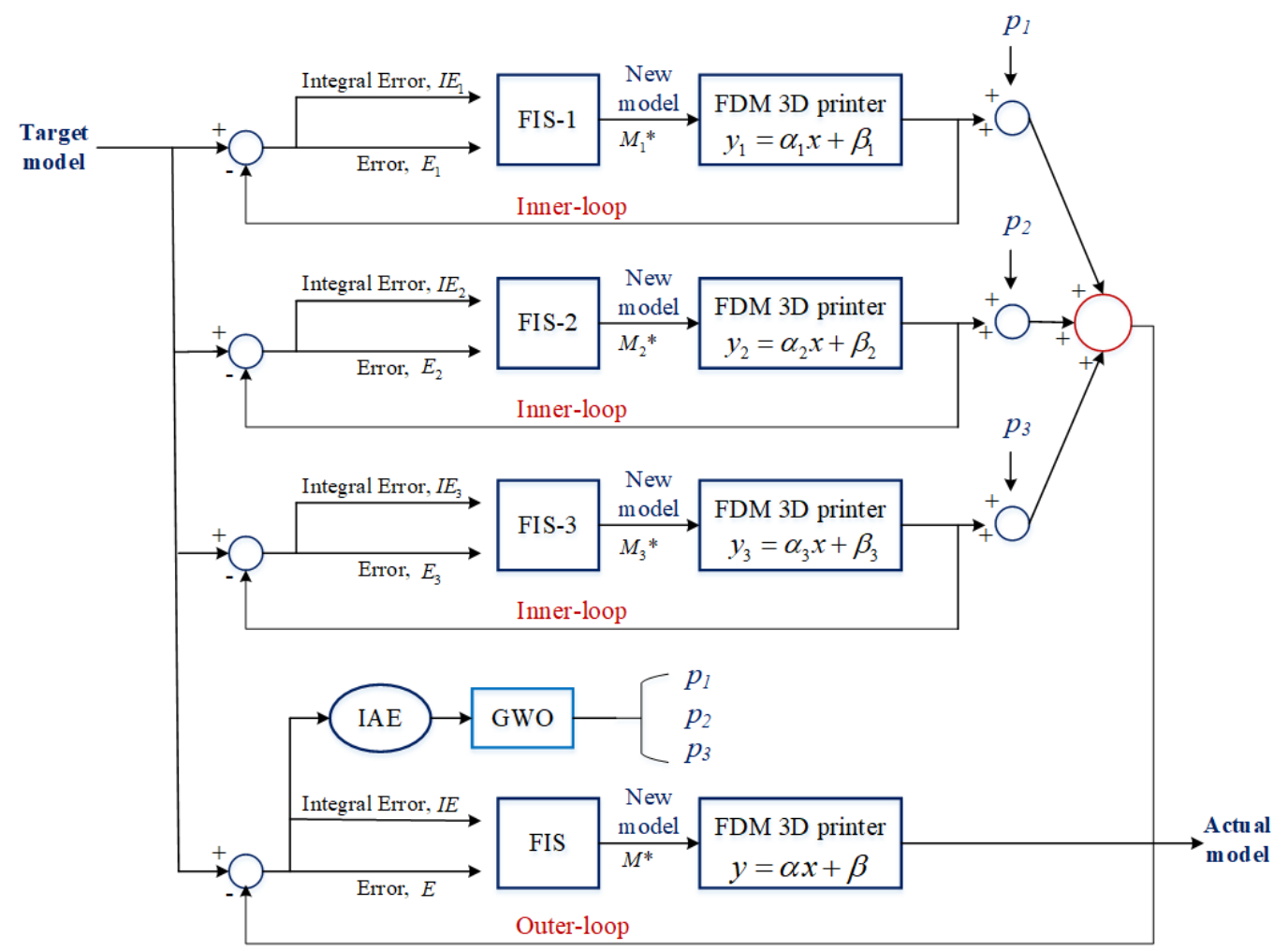

Fig. 4 The error compensation model

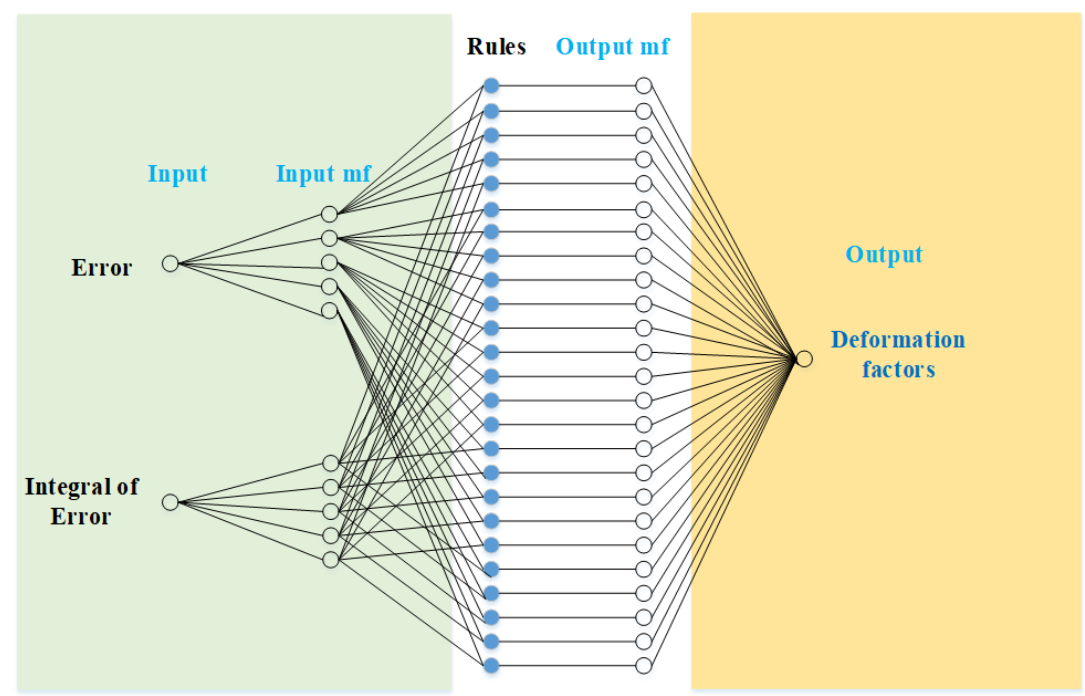

Fig. 5 The FIS structure

It is known that $\alpha, \beta$, and $\delta$ wolves are the three best search agents giving the first, the second, and the third fittest solutions, respectively. However, since an extra emphasis is not given yet for $\alpha$ solution, we introduce an emphasis coefficient in the expression of the search agent positions (Eq. (8)) to influence the other two best solutions. Thus, Eq. (8) can be modified as:

$\mathbf{X}(t+1)=\frac{\eta \mathbf{X}_{1}+\mathbf{X}_{2}+\mathbf{X}_{3}}{3}$

where the term $\eta$ is an emphasis coefficient conventionally given in the interval $[1,2]$.

The GWO algorithm is clearly illustrated in Fig. 6. 


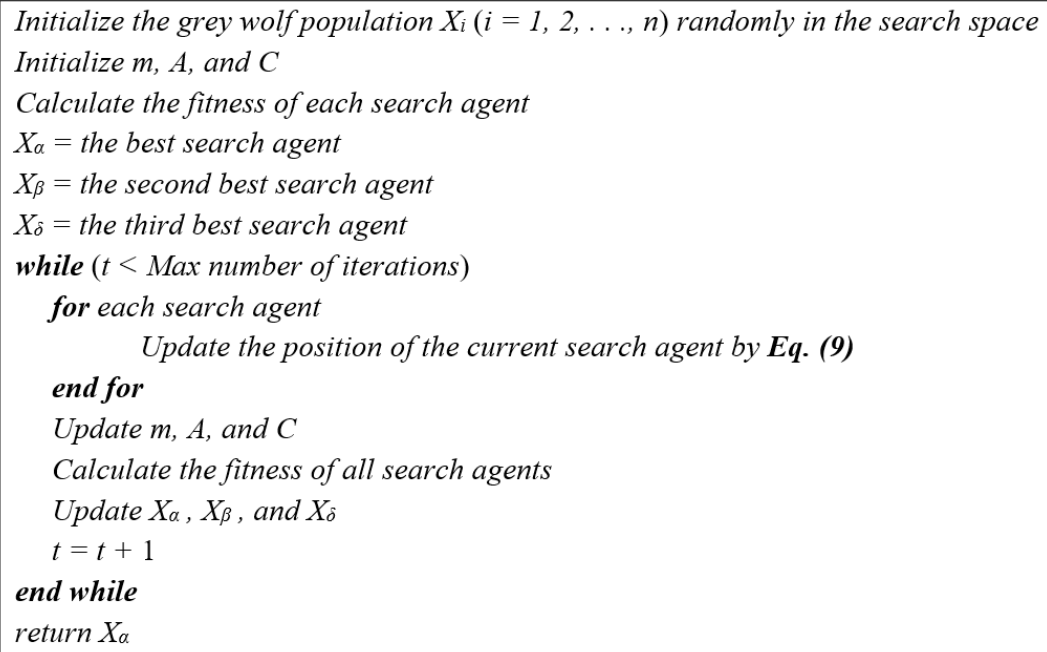

Fig. 6 The GWO algorithm

\section{Results and Discussions}

5.1 The results and discussions of the optimization problem

This section discusses the simulation results of the optimization algorithm that gives the optimal error causing parameters in the three phases of the $3 \mathrm{D}$ printing process (i.e., pre-printing, printing and post-printing). The knowledge of the optimal error causing parameters is useful to make decision which printing stage needs further research for enhancing the print quality. For example, if the possibility of the pre-printing error percentage is more than the other two printing phases, one can decide to do research on a mechanism to reduce the effect of errors associated in the pre-printing phase. And the same scenario applies for the other two cases, printing and post-printing phases.

The GWO algorithm is used to determine the optimal error causing parameters. It applies the following design specifications. The initial position of each wolf is generated based on uniform random distribution. The $\alpha$-wolf's emphasis coefficient is chosen as $\eta=1.2$. Even though it is very difficult to decide the maximum number of iterations and the maximum number of populations for population-based optimization problems [47, we consider nine configuration trials as shown in Table 1. And we choose the one with the best fitness value. Table 2 shows the fitness best solutions for all types of configurations. The integral absolute error is chosen as a standard objective function. It is denoted as a print accuracy index $(P A I)$, which is in fact a metric for our optimization model, and it can be mathematically expressed as:

$P A I=\int_{0}^{t}|e(t)| d t$

where $e(t)$ is the difference between the target model and the actual model.

Our optimization problem is to state the objective function with the possible constraints and solve for the minimum to find the optimal error causing parameters. It is stated as:

$$
\begin{array}{cl}
\text { Min } & P A I=\int_{0}^{t}|e(t)| d t \\
\text { s.t. } & \\
& 0 \leq P_{1} \leq 1 \\
& 0 \leq P_{2} \leq 1 \\
& \leq \leq P_{3} \leq 1
\end{array}
$$

where $P_{1}, P_{2}$ and $P_{3}$ are the three error causing parameters during pre-printing, printing, and post-printing, given in percentage, respectively. The ranges are in the interval $[0,1]$.

Fig. 7] shows the convergence curve of Config-1, Config4 and Config-7. The total number of iterations are taken as 50 for different number of wolves. Config- 4 achieves better fitness solution and higher convergence speed as compared with the other two configurations. Similarly, Fig. 8 presents the convergence curve of Config-2, Config-5 and Config- 8 for 75 number of iterations for different number of wolves. From the three configurations, Config- 5 has better fitness value and higher convergence speed. Config-3, Config- 6 and Config-9 are operated with 100 number of iterations for different number of wolves, which are shown in Fig. 9. The better 
Table 1 List of optimization trials

\begin{tabular}{ccccc}
\hline No. of wolves & No. of iterations & Optimal values, $P_{1}, P_{2}, P_{3}$ & Fitness best solutions & Configuration notations \\
\hline 30 & 50 & $0.2877,0.4854,0.1979$ & 1.8674 & Config-1 \\
30 & 75 & $0.5408,0.1359,0.2039$ & 1.8531 & Config-2 \\
30 & 100 & $0.0493,0.4137,0.4012$ & 1.7579 & Config-3 \\
60 & 50 & $0.2910,0.1278,0.5012$ & 1.7424 & Config-4 \\
60 & 75 & $0.1314,0.2903,0.4843$ & 1.7886 & Config-5 \\
$\mathbf{6 0}$ & $\mathbf{1 0 0}$ & $\mathbf{0 . 3 2 6 0 , 0 . 1 6 6 3 , 0 . 0 4 9 9}$ & $\mathbf{1 . 7 2 1 1}$ & Config-6 \\
90 & 50 & $0.1335,0.5337,0.2904$ & 1.7942 & Config-7 \\
90 & 75 & $0.1973,0.1071,0.1905$ & 1.8377 & Config-8 \\
90 & 100 & $0.5310,0.2841,0.1271$ & 1.7465 & Config-9 \\
\hline
\end{tabular}

Table 2 Fitness best solutions for all configuration types

\begin{tabular}{cccc}
\hline Number of wolves & \multicolumn{3}{c}{ Number of iterations } \\
\cline { 3 - 4 } & 50 & 75 & 100 \\
\hline 30 & 1.8674 & 1.8531 & 1.7579 \\
60 & 1.7424 & 1.7886 & $\mathbf{1 . 7 2 1 1}$ \\
90 & 1.7942 & 1.8377 & 1.7465 \\
\hline
\end{tabular}

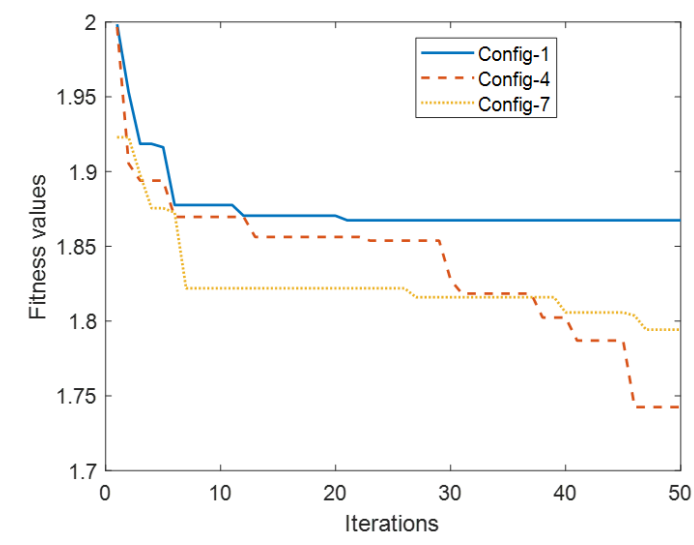

Fig. 7 Convergence curves for Config-1, Config-4, and Config-7

fitness value and higher convergence speed is observed in Config- 6 compared to the rest two configurations.

From the nine configuration trials, it is observed that Config-6 that has 100 number of iterations and 60 wolves achieves the best fitness value and highest convergence speed. The global minimum is observed in the iteration interval $[86,100]$. Therefore, Config- 6 is selected for our optimization problem. With this configuration, the optimal error causing parameters are evaluated as shown in Fig. 10. From the figure, the percentage values for the three parameters i.e., $P_{1}, P_{2}$ and $P_{3}$ are given as $32.6 \%, 16.63 \%$ and $4.99 \%$ respectively. This means that the print quality of the $3 \mathrm{D}$ printing system is affected by the errors associated in $32.6 \%$ during pre-printing phase, $16.63 \%$ during printing phase, $4.99 \%$ during post-printing phase, and the rest is due to external disturbances. The results give us information to decide which phase of the $3 \mathrm{D}$ printing process need further quality monitoring mechanism. In the present

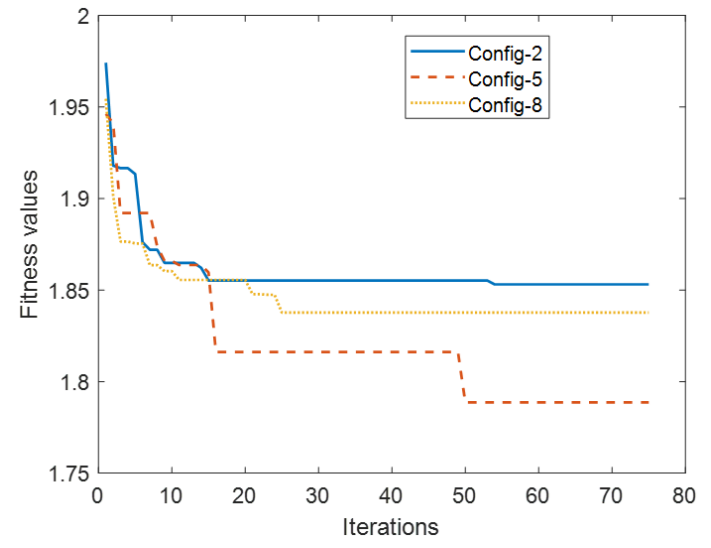

Fig. 8 Convergence curves for Config-2, Config-5, and Config-8

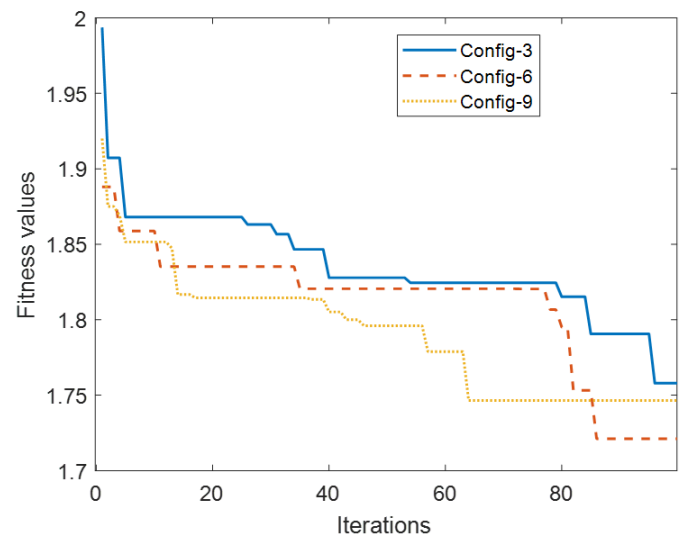

Fig. 9 Convergence curves for Config-3, Config-6, and Config-9

scenario, it is observed that the errors associated in the pre-printing phase have a higher probability to influence the print quality. Although more focus is necessary in the pre-printing phase, it is recommended to investigate a general error compensation framework that considers all the 3D printing phases. A feedback-based fuzzy system is introduced to compensate the effects of errors in the $3 \mathrm{D}$ printing process. The simulation re- 


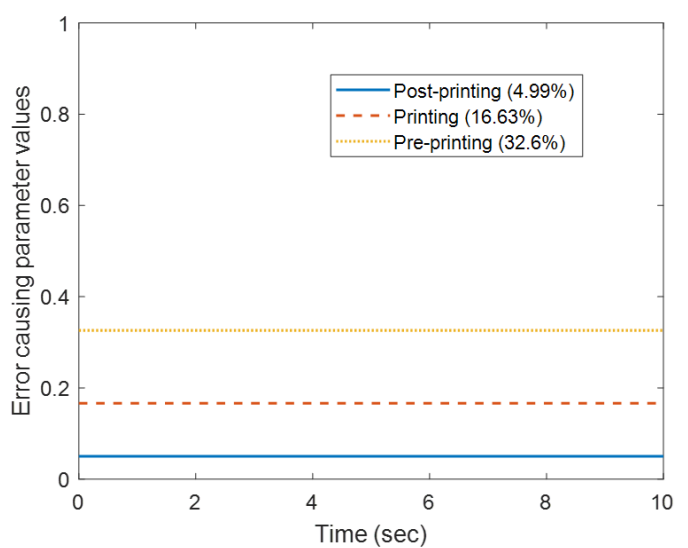

Fig. 10 The error causing parameters; pre-printing, printing and post-printing, given in percentage (\%)

sults of the proposed error compensation mechanism are presented next.

\subsection{The closed-loop results analysis and discussions}

This section discusses the simulation results of the proposed error compensation mechanism. It presents a detailed analysis of the results of the controlling performance of FIS in both the inner-loops and the outerloop. Fig. 11 shows the three deformation factors of the inner-loops. These deformation factors tell us about the reduction or the enlargement of the print object. The deformation factors of all the three inner-loops, i.e., $\alpha_{1}$, $\alpha_{2}$ and $\alpha_{3}$ are computed by setting the range of $\beta_{1}, \beta_{2}$ and $\beta_{3}$ in the interval $[-0.01,0.01]$. Settings of the three $\beta$ ranges are based on conventions in hope of reducing deformation of the print object. The deformation ranges are named as "reduced-large", "reduced-small", "normal", "enlarged-small" and "enlarged-high" whose values in units are set as $[0.4,0.8],[0.8,1.2],[1.2,1.6]$, and $[1.6,2]$, respectively. The error compensation system simulation in Matlab is run for 10 seconds. From Fig. 11. it is observed that after 1.7 seconds the first two deformation factors are approaching to 1 , whereas, the third deformation factor is approaching to 1 after 5.5 seconds. All the three responses are in fact under "normal" deformation range. Fig. 12 shows the model errors of the three inner-loops. It clears that the first two individual errors are approaching to zero after 1.7 seconds, whereas the third individual error is approaching to 1 after 5.5 seconds. Similarly, the deformation factor and the model error of the outer-loop is presented in Fig. 13. It shows that the model error is down to zero after 7 seconds and at the same time the deformation factor is found in the interval $[0.8,1]$, which is under "normal" range and acceptable value.

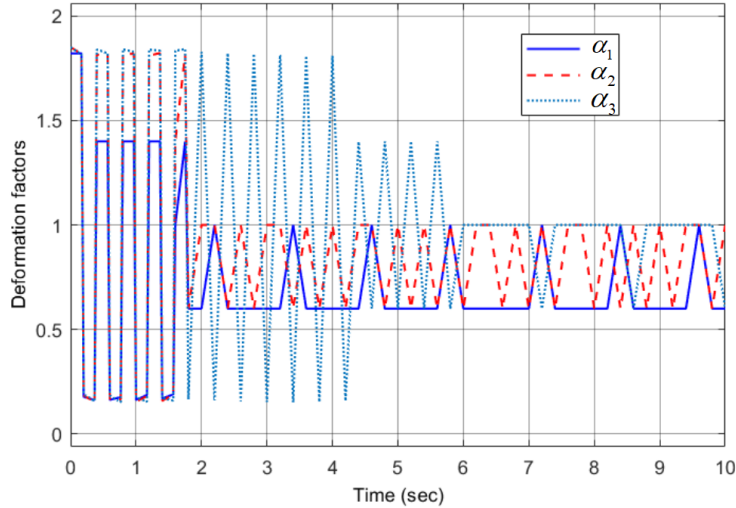

Fig. 11 The deformation factors for the inner-loops

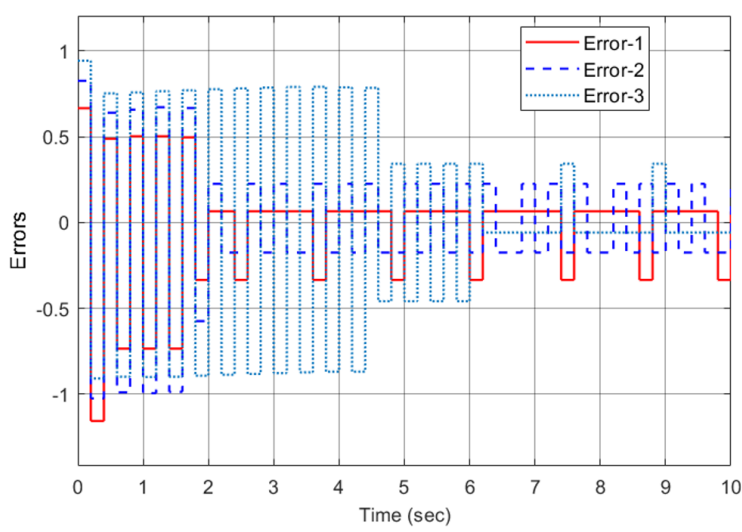

Fig. 12 The model errors for the inner-loops

It is observed that the integration of fuzzy system and GWO algorithm in the 3D printing process gives a promising result by minimizing the effects of the error causing parameters, and hence it improves the print quality. Thus, one can come with an idea that a closedloop-based quality monitoring approach is a viable solution to guarantee the print quality in the $3 \mathrm{D}$ printing process. Last, it can be said that considering all the possible error causing parameters and the nice modelling of the $3 \mathrm{D}$ printing process results in a better control performance. In fact, it is very difficult to explore all the errors associated in the 3D printing process due to a lot of design constraints. And also, removing all the errors at the same time is not a simple task. It is recommended to consider the most influential errors in the print quality and then to design the error compensation framework.

\section{Conclusion}

Knowing the negative effects of printing errors in the quality of the printed object, this paper proposes a 


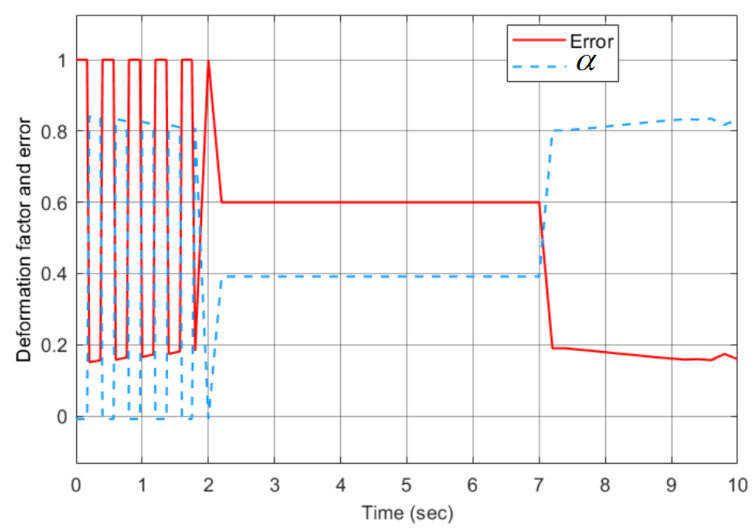

Fig. 13 The deformation factor and the model error for the outer-loop

feedback-based error compensation strategy that considers the three phases of the 3D printing process: preprinting, printing, and post-printing. The objectives of this paper are twofold. 1) To identify the type of errors in every phase of FDM 3D printing. This means that the errors in the three phases of the $3 \mathrm{D}$ printing process are summarized. 2) To propose an end-to-end error compensation strategy by designing a feedback-based fuzzy inference system. The former is used to know the performance of the $3 \mathrm{D}$ printing process in the presence of the possible error causing parameters. The latter is dedicated to determine the optimal error causing parameters by integrating FIS and GWO algorithm. The knowledge of optimal error causing parameters is used to give a decision about which phases of the $3 \mathrm{D}$ printing process need further monitoring to enhance the print quality. For example, if the print quality is possibly affected due to the errors associated in the pre-printing phase, one can decide to make an effort to minimize the errors in this phase, and the same scenario applies for the printing phase and the post-printing phase.

From the nine optimization configurations adopted, Config- 6 that has 60 wolves and 100 number of iterations is selected due to its best fitness value and higher convergence speed. The global minimum is found in the iteration interval $[86,100]$. The proposed feedbackbased fuzzy system has a promising result by generating the deformation factors in a normal range within less than 2 seconds for the inner-loops. An outer-loop generates the deformation factor within 7 seconds. Last, it is observed that the paper demonstrated how a closedloop-based control system is used in 3D printing to minimize the effects of printing errors. However, all the results are simulation-based analysis. The results can be further improved by refining the control and optimization model. The next step is to do a real experimental setups and investigate the performance of the proposed error compensation approach in a real scenario.

\section{Declarations}

Funding This work was supported in part by the National Key Research and Development Program of China (No. 2018YFB1700403); National Natural Science Foundation of China under Grants 61773382, U1909204, 61773381, 61872365 and 61701471; CAS Key Technology Talent Program (Zhen Shen); Hunan Province Science and Technology Program 2018GK1040; Dongguan's Innovation Talents Project (Gang Xiong).

Competing interests The authors declare that they have no competing interests.

Data availability The authors confirm that the data supporting the findings of this study are available within the article.

Code availability The code will be made available on request.

Ethical approval Not applicable.

Consent to participate Not applicable.

Consent to publication Not applicable.

\section{References}

1. C. E. Okwudire and H. V. Madhyastha, "Distributed manufacturing for and by the masses," Science, vol. 372, no. 6540, pp. 341-342, 2021.

2. V. Sharma, H. Roozbahani, M. Alizadeh, and H. Handroos, "3D printing of plant-derived compounds and a proposed nozzle design for the more effective 3D FDM printing," IEEE Access, vol. 9, pp. 57107-57119, 2021.

3. P. K. Penumakala, J. Santo, and A. Thomas, "A critical review on the fused deposition modeling of thermoplastic polymer composites," Composites Part B: Engineering, p. 108336, 2020.

4. M. M. Hoque, M. M. H. Jony, M. M. Hasan, and M. H. Kabir, "Design and implementation of an FDM based 3D printer," in 2019 International Conference on Computer, Communication, Chemical, Materials and Electronic Engineering (IC4ME2). IEEE, 2019, pp. 1-5.

5. Z. Shen, X. Shang, M. Zhao, X. Dong, G. Xiong, and F.-Y. Wang, "A learning-based framework for error compensation in 3D printing," IEEE Transactions on Cybernetics, vol. 49, no. 11, pp. 4042-4050, 2019.

6. Y. Jin, J. Du, Y. He, and G. Fu, "Modeling and process planning for curved layer fused deposition," The International Journal of Advanced Manufacturing Technology, vol. 91 , no. 1 , pp. $273-285,2017$. 
7. T. Chvalina, "7 problems affecting the quality of your 3D prints," https://blog.prusaprinters.org/ 7-problems-affecting-quality-of-3d-prints/, 2018, accessed January 10, 2021.

8. A. Bellini and S. Güçeri, "Mechanical characterization of parts fabricated using fused deposition modeling," Rapid Prototyping Journal, 2003.

9. J. F. Rodrı' guez, J. P. Thomas, and J. E. Renaud, "Design of fused-deposition ABS components for stiffness and strength," J. Mech. Des., vol. 125, no. 3, pp. 545-551, 2003.

10. M. Grieves and J. Vickers, "Digital twin: Mitigating unpredictable, undesirable emergent behavior in complex systems," in Transdisciplinary perspectives on complex systems. Springer, 2017, pp. 85-113.

11. R. P. Rolle, V. de Oliveira Martucci, and E. P. Godoy, "Architecture for digital twin implementation focusing on industry 4.0," IEEE Latin America Transactions, vol. 18, no. 05, pp. 889-898, 2020.

12. A. Fuller, Z. Fan, C. Day, and C. Barlow, "Digital twin: Enabling technologies, challenges and open research," IEEE Access, vol. 8, pp. $108952-108971,2020$.

13. C. Liu, A. C. C. Law, D. Roberson, and Z. J. Kong, "Image analysis-based closed loop quality control for additive manufacturing with fused filament fabrication," Journal of Manufacturing Systems, vol. 51, pp. 75-86, 2019.

14. M. Faes, W. Abbeloos, F. Vogeler, H. Valkenaers, K. Coppens, T. Goedemé, and E. Ferraris, "Process monitoring of extrusion based 3D printing via laser scanning," arXiv preprint arXiv:1612.02219, 2016.

15. D. Ikeuchi, A. Vargas-Uscategui, X. Wu, and P. C. King, "Data-efficient neural network for track profile modelling in cold spray additive manufacturing," Applied Sciences, vol. 11 , no. 4, p. 1654, 2021.

16. A. Saluja, J. Xie, and K. Fayazbakhsh, "A closed-loop in-process warping detection system for fused filament fabrication using convolutional neural networks," Journal of Manufacturing Processes, vol. 58, pp. 407-415, 2020.

17. A. Cerro, P. E. Romero, O. Yiğit, and A. Bustillo, "Use of machine learning algorithms for surface roughness prediction of printed parts in polyvinyl butyral via fused deposition modeling," The International Journal of Advanced Manufacturing Technology, pp. 1-11, 2021.

18. Y. Fu, A. Downey, L. Yuan, A. Pratt, and Y. Balogun, "In situ monitoring for fused filament fabrication process: A review," Additive Manufacturing, p. 101749, 2020.

19. A. Oleff, B. Küster, M. Stonis, and L. Overmeyer, "Process monitoring for material extrusion additive manufacturing: a state-of-the-art review," Progress in Additive Manufacturing, pp. 1-26, 2021.

20. L. A. Zadeh, "Fuzzy sets," Information and Control, vol. 8, no. 3, pp. 338-353, 1965.

21. H.-C. Liu, X. Luan, M. Zhou, and Y. Xiong, "A new linguistic petri net for complex knowledge representation and reasoning," IEEE Transactions on Knowledge and Data Engineering, 2020.

22. C. Wang, W. Pedrycz, J. Yang, M. Zhou, and Z. Li, "Wavelet frame-based fuzzy C-means clustering for segmenting images on graphs," IEEE Transactions on Cybernetics, vol. 50, no. 9, pp. 3938-3949, 2019.

23. F. Li, T. W. Liao, W. Cai, and L. Zhang, "Multitask scheduling in consideration of fuzzy uncertainty of multiple criteria in service-oriented manufacturing," IEEE Transactions on Fuzzy Systems, vol. 28, no. 11, pp. 27592771, 2020.

24. L. Wang, W. Dai, J. Ai, W. Duan, and Y. Zhao, "Reliability evaluation for manufacturing system based on dynamic adaptive fuzzy reasoning petri net," IEEE Access, vol. 8, pp. $167276-167287,2020$.

25. S. Mirjalili, S. M. Mirjalili, and A. Lewis, "Grey wolf optimizer," Advances in Engineering software, vol. 69, pp. 46-61, 2014.

26. S. N. Ghorpade, M. Zennaro, and B. S. Chaudhari, "GWO model for optimal localization of IoT-enabled sensor nodes in smart parking systems," IEEE Transactions on Intelligent Transportation Systems, 2020.

27. F. Yan, X. Xu, and J. Xu, "Grey wolf optimizer with a novel weighted distance for global optimization," IEEE Access, vol. 8, pp. $120173-120197,2020$.

28. W. Cao and Y. Miyamoto, "Direct slicing from AutoCAD solid models for rapid prototyping," The International Journal of Advanced Manufacturing Technology, vol. 21, no. 10-11, pp. 739-742, 2003.

29. J. Feng, J. Fu, Z. Lin, C. Shang, and B. Li, "Direct slicing of T-spline surfaces for additive manufacturing," Rapid Prototyping Journal, 2018.

30. H. Mao, T.-H. Kwok, Y. Chen, and C. C. Wang, "Adaptive slicing based on efficient profile analysis," ComputerAided Design, vol. 107, pp. 89-101, 2019.

31. Y. Garashchenko and N. Zubkova, "Adaptive slicing in the additive manufacturing process using the statistical layered analysis," in Design, Simulation, Manufacturing: The Innovation Exchange. Springer, 2020, pp. 253-263.

32. J. Jiang, C. Yu, X. Xu, Y. Ma, and J. Liu, "Achieving better connections between deposited lines in additive manufacturing via machine learning," Math. Biosci. Eng, vol. 17 , no. $4,2020$.

33. Z. Li, Z. Zhang, J. Shi, and D. Wu, "Prediction of surface roughness in extrusion-based additive manufacturing with machine learning," Robotics and ComputerIntegrated Manufacturing, vol. 57, pp. 488-495, 2019.

34. M. Khanzadeh, S. Chowdhury, M. Marufuzzaman, M. A. Tschopp, and L. Bian, "Porosity prediction: supervisedlearning of thermal history for direct laser deposition," Journal of Manufacturing Systems, vol. 47, pp. 69-82, 2018.

35. L. Frick, "How to avoid errors during desktop 3D printing," https://www. machinedesign.com/3d-printing-cad/article/21831695/ how-to-avoid-errors-during-desktop-3d-printing, 2013, accessed June 20, 2021.

36. Z. Pilch, J. Domin, and A. Szłapa, "The impact of vibration of the 3D printer table on the quality of print," in 2015 Selected Problems of Electrical Engineering and Electronics (WZEE). IEEE, 2015, pp. 1-6.

37. H. Zhang, W. Zhong, Q. Hu, M. Aburaia, J. GonzalezGutierrez, and H. Lammer, "Research and implementation of axial 3D printing method for PLA pipes," Applied Sciences, vol. 10, no. 13, p. 4680, 2020.

38. G. Zhao, G. Ma, J. Feng, and W. Xiao, "Nonplanar slicing and path generation methods for robotic additive manufacturing," The International Journal of Advanced Manufacturing Technology, vol. 96, no. 9, pp. 3149-3159, 2018.

39. D. Ahlers, F. Wasserfall, N. Hendrich, and J. Zhang, "3D printing of nonplanar layers for smooth surface generation," in 2019 IEEE 15th International Conference on Automation Science and Engineering (CASE). IEEE, 2019, pp. 1737-1743.

40. C. Qingquan, Y. Xunhe, and N. Jing, "Design and optimization of heat dissipation and cooling device for the open 3D printer," in 2020 Chinese Control And Decision Conference (CCDC). IEEE, 2020, pp. 814-819. 
41. T. D. Ngo, A. Kashani, G. Imbalzano, K. T. Nguyen, and D. Hui, "Additive manufacturing (3D printing): A review of materials, methods, applications and challenges," Composites Part B: Engineering, vol. 143, pp. 172-196, 2018.

42. N. Van de Werken, H. Tekinalp, P. Khanbolouki, S. Ozcan, A. Williams, and M. Tehrani, "Additively manufactured carbon fiber-reinforced composites: State of the art and perspective," Additive Manufacturing, vol. 31, p. 100962, 2020.

43. A. N. Dickson, H. M. Abourayana, and D. P. Dowling, "3D printing of fibre-reinforced thermoplastic composites using fused filament fabrication-A review," Polymers, vol. 12 , no. 10 , p. $2188,2020$.

44. N. Krajangsawasdi, L. G. Blok, I. Hamerton, M. L. Longana, B. K. Woods, and D. S. Ivanov, "Fused deposition modelling of fibre reinforced polymer composites: a parametric review," Journal of Composites Science, vol. 5, no. 1, p. 29, 2021.

45. P. J. Prieto-Entenza, N. R. Cazarez-Castro, L. T. Aguilar, S. L. Cardenas-Maciel, and J. A. LopezRenteria, "A lyapunov analysis for mamdani type fuzzybased sliding mode control," IEEE Transactions on Fuzzy Systems, vol. 28, no. 8, pp. 1887-1895, 2019.

46. K. Cui, X. Shang, C. Luo, Z. Shen, H. Gao, and G. Xiong, "A kind of accuracy improving method based on error analysis and feedback for DLP 3D printing," in 2019 IEEE International Conference on Service Operations and Logistics, and Informatics (SOLI). IEEE, 2019, pp. 5-9.

47. A. P. Engelbrecht, "Fitness function evaluations: A fair stopping condition?" in 2014 IEEE Symposium on Swarm Intelligence. IEEE, 2014, pp. 1-8. 